\title{
He abundance in Planetary Nebulae
}

\author{
R. Gruenwald and S. M. Viegas \\ IAG-USP; Av. Miguel Stefano, 4200; 04301-904 São Paulo, SP, Brazil
}

\begin{abstract}
Type I planetary nebulae (PNe), defined as those with high $\mathrm{He}$ (and N) abundances, generally present bipolar geometries and high stellar temperatures. The main goal of this paper is to check if the empirically derived He overabundance for Type I PNs is real, or if it is a consequence of geometrical effects due to the bipolarity and/or to the ionization stratification due to the high stellar temperature of these objects.
\end{abstract}

\section{Introduction}

The determination of the chemical abundance in PNe provides information on the products of stellar evolution, as well as on the enrichment of the interstellar medium, establishing constraints for stellar evolutionary models as well as for the study of the chemical evolution of galaxies. Chemical abundances in PNe are usually obtained using empirical methods (as for example in Peimbert \& Torres-Peimbert 1987). Photoionization models also provide the gas chemical composition when applied to specific nebulae (for example, Harrington et al. 1982; Gruenwald, Viegas \& Broguière 1997). Empirical methods, using a few bright emission-lines, are commonly used since they can easily be applied to a great number of objects. Following their abundance, PN are classified in types (Peimbert 1978; Peimbert \& Torres-Peimbert 1983). In particular, Type I PNe are those with high $\mathrm{He}$ and $\mathrm{N}$ abundances, e.g. $\mathrm{He} / \mathrm{H} \geq 0.125$ and $\log (\mathrm{N} / \mathrm{O})$ $>-0.30$. In a study of morphological and physical properties of PNe, Corradi \& Schwarz (1995) stated that bipolar nebulae have the hottest stars among $\mathrm{PNe}$, and, except for two objects, all bipolar PNe for which chemical data are available are Type I. Therefore, objects with high He and $\mathrm{N}$ abundances have bipolar morphologies and high stellar temperatures. However, new evolutionary models fail to explain the high abundance of $\mathrm{He}$ and $\mathrm{N}$ in Type I PNe (van den Hoek \& Groenewegen 1997). In this paper we analyze the effect of the geometry and of the stellar temperature on the empirical abundance determinations, in order to verify if the high abundances of He obtained for Type I PNe are real. For this, we apply our self-consistent 3D photoionization code (Gruenwald et al. 1997) to simulate planetary nebulae.

\section{Method and results}

For a wide range of nebular and stellar characteristics, we obtain the physical conditions in each point of the nebula, and calculate the resulting line intensity 


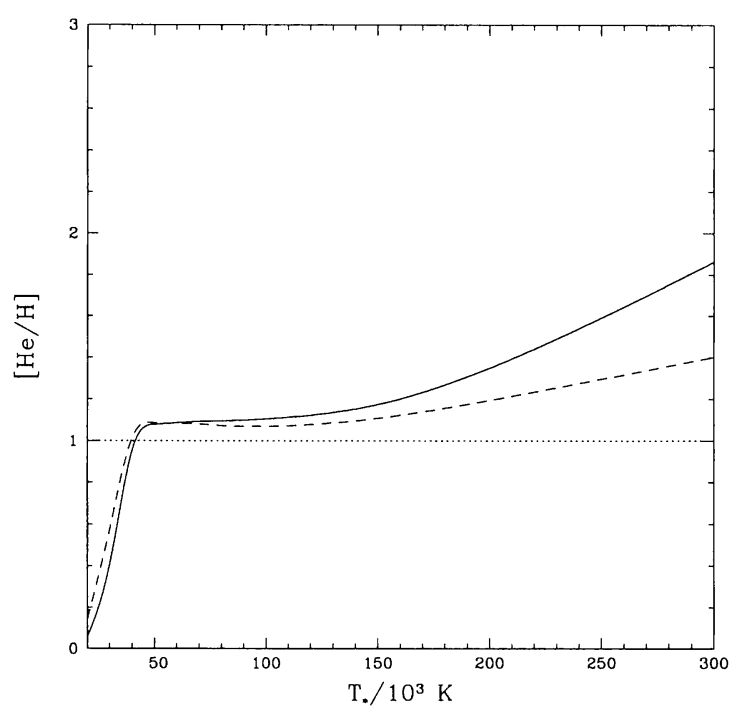

Figure 1. Fig. 1 - Ratio between the empirical and the adopted values for $\mathrm{He} / \mathrm{H}$ versus the stellar temperature. The curves correspond to a nebula with uniform density $\left(\mathrm{n}_{H}=100 \mathrm{~cm}^{-3}, \mathrm{~L}_{*}=3000 \mathrm{~L}_{\odot}\right)$. The solid line represents the results obtained from line intensities corresponding to the whole nebula, while the dashed line corresponds to the results from a narrow slit crossing the nebula through its center.

ratios at different lines of sight to the nebula. From the calculated line intensity ratios we derive the abundances applying the empirical methods used in the literature. These abundances are then compared to those assumed in the models. The effect of the central stellar temperature can be seen in Figure 1. For low stellar temperatures $\left(\mathrm{T}_{*}<510^{4} \mathrm{~K}\right)$, the empirical $\mathrm{He} / \mathrm{H}$ abundance ratio is lower than the real value, while for higher $\mathrm{T}_{*}\left(>10^{5} \mathrm{~K}\right)$ the opposite is true. This is due to the fact that the ionic distribution inside the nebula depends on $\mathrm{T}_{*}$; for example, for low stellar temperatures the $\mathrm{He}^{++}$zone is much smaller than the $\mathrm{H}^{+}$zone; for increasingly temperatures the $\mathrm{He}^{++}$zone is relatively larger. A further consequence is that the obtained abundance depends also on the size and position of the slit used for the observation. In order to check for a geometrical effect, models for bipolar nebulae were also obtained, assuming a spherically symmetrical cloud with an equatorial torus of denser gas close to the ionizing star. Our results show that the abundance obtained from empirical methods can mimic an overabundance and/or the presence of abundance gradients of $\mathrm{He}$ in planetary nebulae with high stellar temperature. Geometrical effects strengthen this problem. 


\section{References}

Corradi, R.L.M., \& Schwarz, H.E. 1995, A\&A, 293, 871

Gruenwald, R., Viegas, S.M., \& Broguiere, D. 1997, ApJ, 480, 283

Harrington, J.P. et al. 1982, MNRAS, 199,517

Peimbert 1978, in IAU Symp. 76, Planetary Nebulae, ed. Y. Terzian (Dordrecht: Reidel), 215

Peimbert, M. \& Torres-Peimbert, S. 1983, in IAU Symp. 76, Planetary Nebulae, ed. D.R. Flower (Dordrecht:Kluwer),233

Peimbert, M. \& Torres-Peimbert, S. 1987, Rev.Mex.Astr.Astroph., 14, 540

van den Hoek, L.B. \& Groenewegen, M.A.T. 1997, A\&AS, 123, 305 